\title{
SARS-CoV-2 infection in the Italian Veneto region: adverse outcomes in patients with cancer
}

\author{
Massimo Rugge $\oplus^{1,2 \bowtie}$, Manuel Zorzi ${ }^{2}$ and Stefano Guzzinati ${ }^{2}$
}

\begin{abstract}
In 84,246 consecutive Italians tested for SARS-CoV-2, we examined the prevalence of cancer and clinical outcomes of viral infection. Overall, $5.7 \%(4,789 / 84,246)$ had previously been diagnosed with cancer and, among the SARS-CoV2-positive (CV2+ve) patients, $7.8 \%$ had a cancer diagnosis $(723 / 9,275)$. Prevalence of cancer was not associated with risk of infection (odds ratio of $0.97 ; 95 \%$ confidence interval of 0.89-1.06). The proportion of CV2+ve people hospitalized was higher among patients with cancer ( 56.6 versus $34.4 \%$ among other people), and so was the proportion of deaths (14.7\% versus $4.5 \%)$. The risk of adverse outcomes of SARS-CoV-2 infection was significantly higher for patients with cancer versus those without, particularly for males and older people.
\end{abstract}

On 31 December 2019, the World Health Organization (WHO) was informed about a cluster of cases of a worrying infectious pneumonia of unknown etiology in Wuhan, Hubei Province, China. Three days later, 11 of the 44 initially reported infected individuals were reportedly as severely ill. The infectious disease was called novel coronavirus 2019.

The infectious agent was subsequently detected in Italy (31 January 2020) in two Chinese patients, and then the number of individuals infected rose rapidly to 1,694 by 1 March (https:// www.epicentro.iss.it/coronavirus/sars-cov-2-sorveglianza-dati; accessed 24 April 2020). On 11 March, the WHO Director-General said, "We have therefore made the assessment that COVID-19 can be characterized as a pandemic" (https://www.who.int/dg/ speeches/detail/who-director-general-s-opening-remarks-a t-the-media-briefing-on-covid-19-11-march-2020; accessed 24 April 2020). The clinical course of SARS-CoV-2 infection has yet to be fully characterized, and the clinical syndrome can range from asymptomatic infection to severe acute respiratory distress syndrome, even progressing to multi-organ failure.

Because of their particular clinical and biological risk factors (systemic immunosuppression, surgical treatments and/or chemotherapy), patients with cancer (irrespective of the primary site and clinical stage of their disease) are plausibly considered more susceptible to infection than individuals without cancer ${ }^{1-5}$.

This report addresses the prevalence of patients with a clinical history of cancer in an Italian population tested for SARS-CoV-2 infection among the residents of the Veneto region in the northeast of Italy (with an overall population of 4,906,000 in 2019).

The primary outcomes of this study were: (1) a quantification of the prevalence of patients with a history of cancer in the population tested for SARS-CoV-2 infection and (2) an estimation of the association between a history of cancer and clinical outcomes of patients who were CV2+ve.

\section{Results}

Of the 84,246 individuals tested, 4,789 (5.7\%) had a history of cancer (Table 1). The proportion was higher for males $(2,311 / 33,183$ $(7.0 \%))$ than for females $(2,478 / 51,063(4.9 \%))$.

When the individuals tested for SARS-CoV-2 infection were stratified by age (under 70 years old, 71,100; 70-79years old, 5,$551 ; 80$ or more years old, 7,595), the prevalence of CV2+ve patients with cancer was $281 / 2,628(10.7 \%), 186 / 1,025(18.1 \%)$ and $256 / 1,136(22.5 \%)$, respectively, in the three age categories (test for trend $P<0.0001)$.

Using logistic regression analysis, the prevalence odds ratio (pOR) of a CV2+ve status in the study population was statistically associated with sex (female versus male, $\mathrm{pOR}=0.68,95 \%$ confidence interval $(\mathrm{CI})=0.65-0.71)$ and age $(70-79$ versus $0-69$ years, $\mathrm{pOR}=2.73,95 \% \mathrm{CI}=2.55-2.93$; $\geq 80$ years versus $0-69$ years, $\mathrm{pOR}=3.15,95 \% \mathrm{CI}=2.96-3.34)$. A clinical history of cancer was not associated with CV2+ve status ( $\mathrm{pOR}=0.97,95 \% \mathrm{CI}=0.89-1.06$ ).

Table 1 shows the demographics for the CV2+ve individuals (patients with cancer versus those without): 723/9,275 (7.8\%) had been diagnosed with cancer. Males prevailed $(P=0.0025)$ among these patients with cancer, and their distribution increased with older age (test for trend $P<0.0001$ ). Among the CV2+ve individuals needing to be hospitalized, a statistically significant difference between the patients with cancer and the others emerged only for age (test for trend $P<0.0001$ ).

Among the $723 \mathrm{CV} 2+$ ve patients with cancer, 55 (7.6\%) had a history of multiple malignancies $(\mathrm{M} / \mathrm{F}=38: 17) ; 35$ of these 55 patients $(63.6 \%)$ were hospitalized due to their SARS-CoV-2 infection and 8/55 (14.5\%) died (results not shown in the table).

Table 1 also shows the prevalence of clinical outcomes among the $723 \mathrm{CV} 2+$ ve patients with cancer and the other 8,552 (patients who did not have cancer) CV2+ve individuals, overall and by sex. The proportion of CV2+ve individuals hospitalized was $56.6 \%$ (409/723) among patients with cancer, as compared with $34.4 \%$ $(2,941 / 8,552)$ among the others $(P<0.0001)$. The prevalence of those admitted to the intensive care unit (ICU) due to SARS-CoV2-related disease did not differ between the patients with cancer and the others ( $7.5 \%$ versus $6.2 \%, P=0.18$ ). Of the $723 \mathrm{CV} 2+$ ve patients with cancer, 106 died of SARS-CoV-2-related disease during the study period (14.7\%), as compared with 385 of $8,552(4.5 \%)$ of the other CV2+ve individuals $(P<0.0001)$.

Using logistic regression (pOR adjusted for sex and age), the risk of hospitalization and death was consistently lower among females $(\mathrm{POR}=0.35,95 \% \mathrm{CI}=0.28-0.38$ and $\mathrm{POR}=0.44,95 \% \mathrm{CI}=0.36-$ 0.54, respectively; Fig. 1). Compared to younger people, CV2+ve patients aged 70 years or more were at greater risk of hospitalization $(\mathrm{pOR}=4.02)$ and death $(\mathrm{pOR}=25.43)$ (Fig. 1).

'Department of Medicine, Surgical Pathology and Cytopathology Unit, University of Padova, Padova, Italy. ${ }^{2}$ Veneto Tumor Registry (RTV), Azienda Zero,

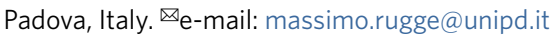


Table 1 | Prevalence of SARS-CoV-2 among 84,246 residents of the Veneto region of northeastern Italy by history of cancer, sex and age

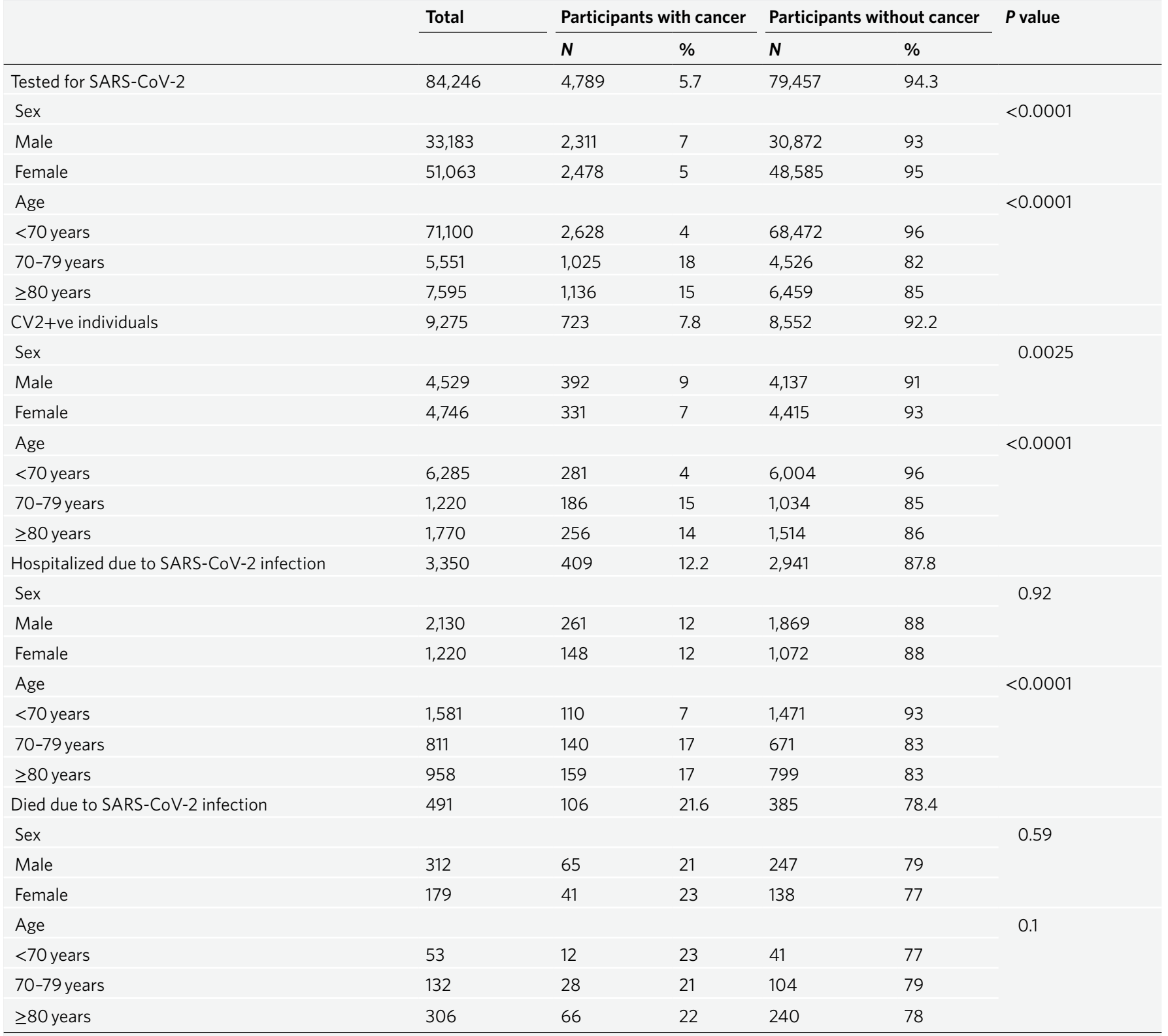

Pearson's chi-square test was used to identify differences in the proportions of individuals between two categories. The Cochran-Armitage test was used to look for any trends for an ordinal variable with more than two categories. Both tests were two-tailed.

As for the interval between cancer diagnosis and infection, individuals who had been diagnosed with cancer within the 2 years before acquiring the infection showed the highest risk of both hospitalization and death $(\mathrm{pOR}=2.67,95 \% \mathrm{CI} 1.74-4)$. A lower, but still significant increased risk was also documented for patients who developed cancer in the previous $2-5$ years $(\mathrm{pOR}=1.68,95 \% \mathrm{CI}$ $1.11-2.48$ ), or more than 5 years before the infection ( $\mathrm{pOR}=1.59$, 95\% CI 1.08-2.31) (Fig. 1). The difference in pORs for death (by latency times between cancer diagnosis and viral infection) was not significant $(P$ value for Wald statistic, 0.10$)$. No statistically significant association emerged between age or history of cancer and admission to the ICU, while such an event was less frequent among women (Fig. 1).

Among CV2+ve patients with cancer, the risk of adverse events was distinguished by site of primary cancer (Table 2), the most common being cases of breast $(n=128)$, prostate $(110)$, colon or rectum
(95) and hematological malignancies (81). A statistically significant higher pOR (after adjusting for sex and age) for hospitalization due to the viral infection emerged for breast, hematological and urological malignancies. As for admission to the ICU, no statistically significant increase in pOR was seen for any of the malignancies considered. A statistically significant higher pOR for death was associated with lung cancer, breast cancer and hematological malignancies.

\section{Discussion}

A recent position paper published in Nature Medicine ("Caring for patients with cancer in the COVID-19 era") stated that "At present, the uncertainty of the true incidence of COVID-19 (symptomatic cases plus asymptomatic cases) makes it impossible to accurately calculate a patient's risk of COVID-19. Determining the incidence of COVID-19 through the use of large-scale serological testing is therefore a priority" 6 . 
a

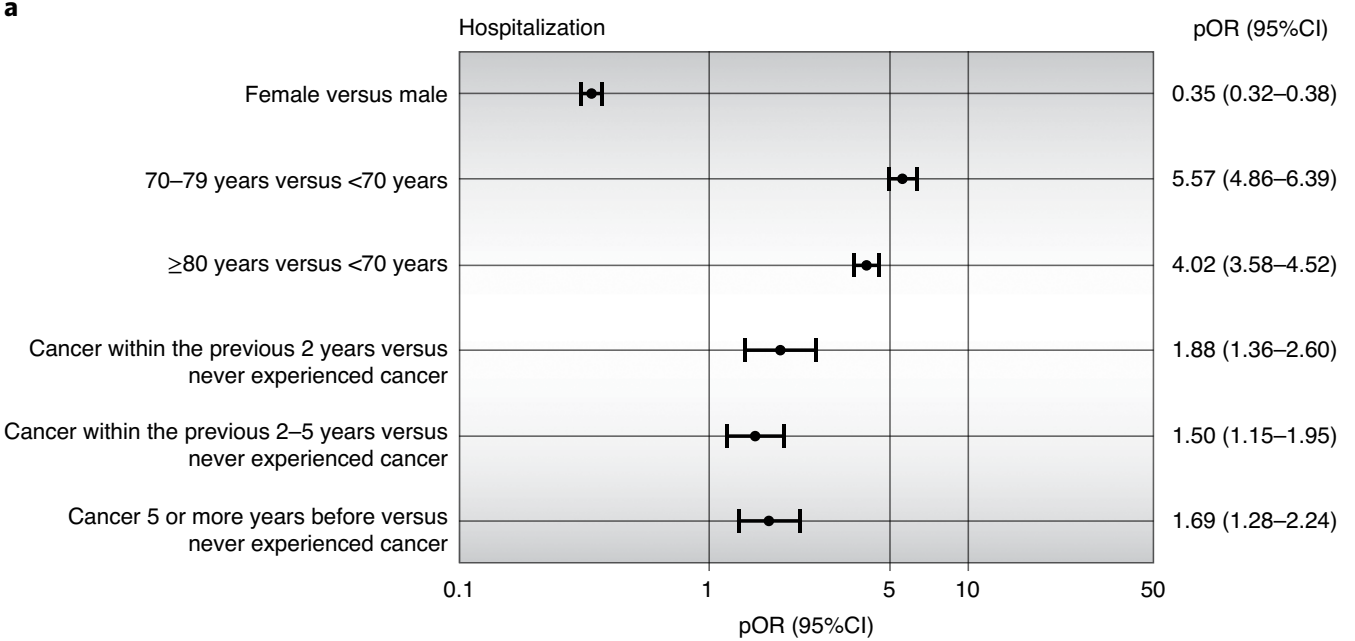

b

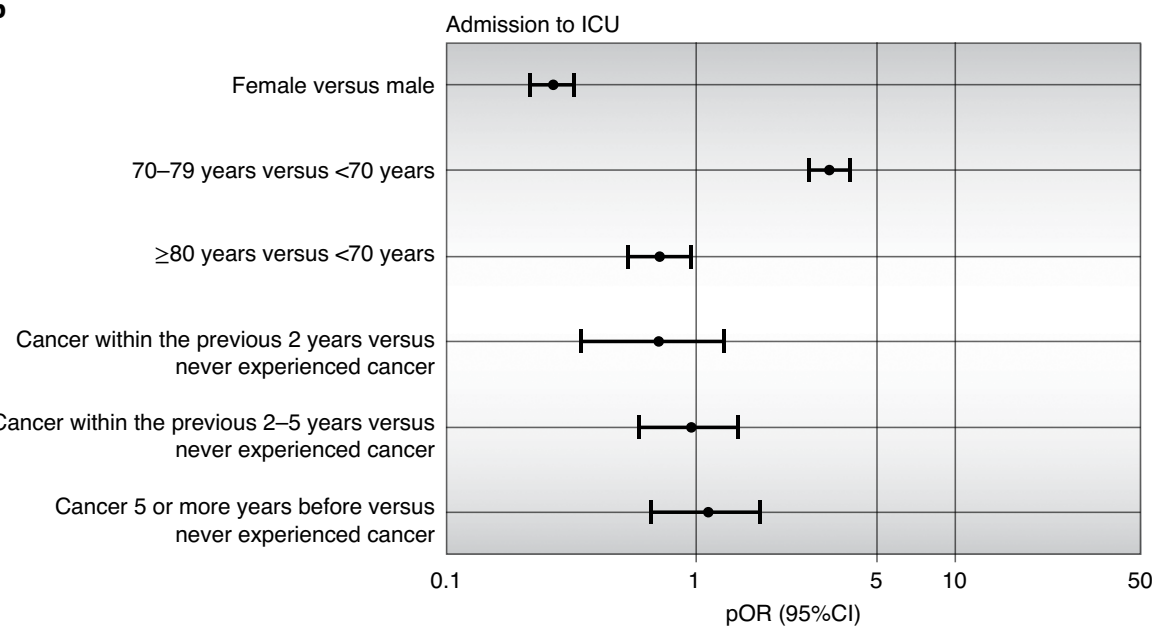

pOR $(95 \% \mathrm{Cl})$

$0.28(0.22-0.34)$

3.27 (2.69-3.97)

$0.72(0.54-0.95)$

$0.71(0.36-1.28)$

$0.95(0.59-1.47)$

$1.11(0.67-1.77)$

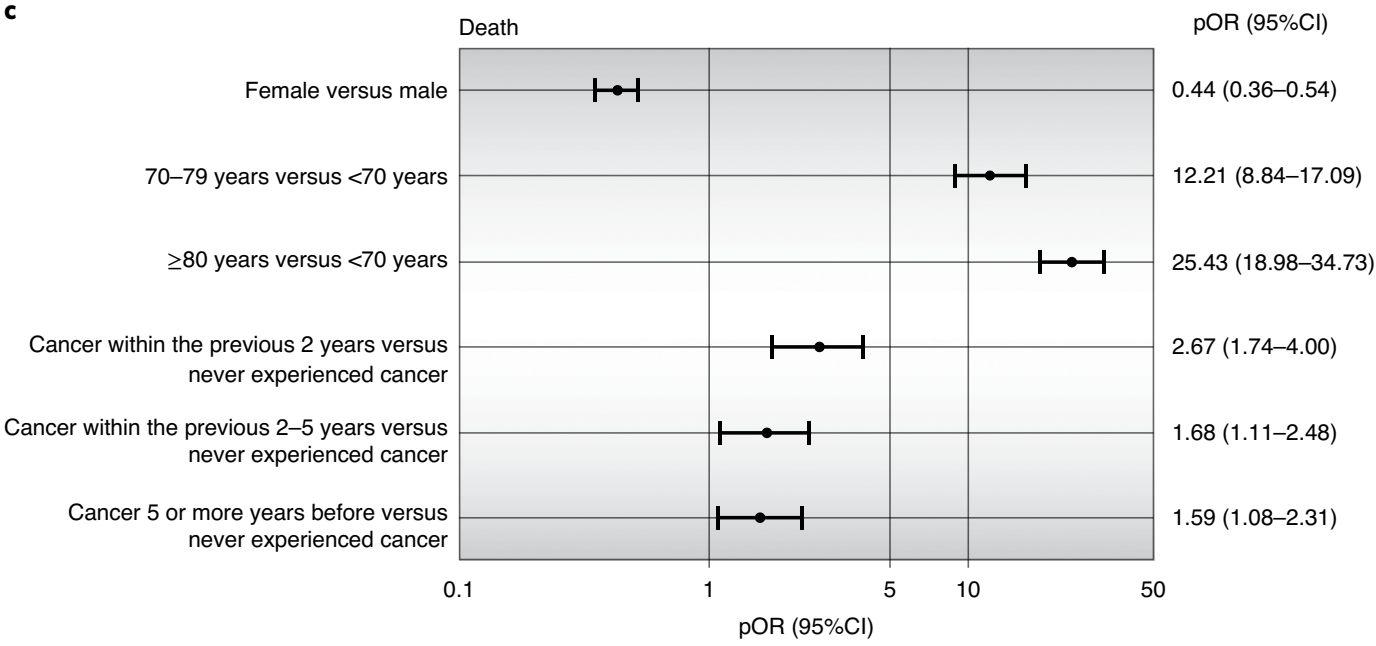

Fig. 1 | Results of the logistic regression on CV2+ve patients. a-c, The data depict the pOR adjusted for sex and age, with error bars reporting $95 \%$ $\mathrm{Cl}$ ( $n=9,275$ patients). The risk of hospitalization (a) and death (c) was consistently lower among females. Compared to younger people, $\mathrm{CV} 2+\mathrm{ve}$ patients aged 70 years or more were at greater risk of hospitalization (a) and death (c). A statistically significant increase in the risk was documented for individuals who had been diagnosed with cancer within the 2 years before acquiring the infection (a,c). No statistically significant association emerged between age or history of cancer and admission to the ICU (b).

The present study addresses the prevalence of individuals with a history of cancer (4,789 participants, 5.7\%) in a large population of 84,246 consecutively tested for SARS-CoV-2 infection.
In the Italian population considered here, individuals with a history of cancer did not show any increased risk associated with $\mathrm{CV} 2+\mathrm{ve}$ status $(\mathrm{pOR}=0.97)$. This apparent discrepancy with 
Table 2 | pOR of cancer by clinical outcome (hospitalization or admission to the ICU versus no hospitalization) or death (dead versus alive)

\begin{tabular}{|c|c|c|c|c|c|c|c|c|c|c|c|c|c|}
\hline \multirow[t]{2}{*}{ Malignancy } & \multirow{2}{*}{$\begin{array}{r}\text { CV2+ve } \\
\text { (number) }\end{array}$} & \multicolumn{4}{|c|}{ Hospitalization } & \multicolumn{4}{|c|}{ Admission to ICU } & \multicolumn{4}{|c|}{ Death } \\
\hline & & $N$ & $\mathrm{pOR}^{\mathrm{a}}$ & & $\% \mathrm{Cl}$ & $N$ & $\mathrm{pOR}^{\mathrm{a}}$ & & $\% \mathrm{Cl}$ & $N$ & $\mathrm{pOR}^{\mathrm{a}}$ & & $\% \mathrm{Cl}$ \\
\hline Prostate $^{c}$ & 110 & 73 & 1.44 & 0.94 & 2.21 & 12 & 0.80 & 0.43 & 1.49 & 15 & 1.25 & 0.70 & 2.25 \\
\hline Colon or rectum & 95 & 52 & 1.19 & 0.77 & 1.85 & 12 & 1.89 & 0.99 & 3.61 & 17 & 1.71 & 0.97 & 3.01 \\
\hline Hematology & 81 & 48 & 2.00 & 1.22 & 3.28 & 3 & 0.40 & 0.12 & 1.31 & 13 & 2.39 & 1.24 & 4.58 \\
\hline Lung & 21 & 13 & 2.04 & 0.77 & 5.42 & 1 & 0.48 & 0.06 & 3.71 & 5 & 3.90 & 1.27 & 11.95 \\
\hline Others & 210 & 103 & 1.47 & 1.09 & 2.00 & 11 & 0.71 & 0.38 & 1.34 & 21 & 1.48 & 0.90 & 2.42 \\
\hline
\end{tabular}

${ }^{\mathrm{a}} \mathrm{pOR}$ is derived from multivariable logistic regression model that includes sex and age in three categories. ${ }^{\mathrm{b}}$ Women only. ${ }^{\mathrm{c}}$ Men only.

respect to clinical expectations $s^{6,7}$ and findings in the earlier-affected Chinese population ${ }^{8-10}$ could be at least partially explained by the demographic profile of the cancer population considered, which mostly included older males.

Consistently with Xia et al. ${ }^{9}$, we support the need to seek further confirmation of our findings due to the possibility of confounding factors resulting from the changing profile of the populations tested over the study period ${ }^{11,12}$.

As expected, the prevalence of SARS-CoV-2 infection increased with age in our sample (with and without a history of cancer) and was higher in males. Similar results emerged in the Chinese population $^{8,13}$.

Focusing on adverse clinical outcomes, CV2+ve males showed a statistically significant higher risk of hospitalization, admission to the ICU, and death. Irrespective of sex, older CV2+ve individuals were also at an up to 25 -fold greater risk of death due to the viral disease. The poor outcome of CV2+ve patients with cancer is consistent with that reported by Zhang in 28 patients with cancer $^{14}$, as well as with the higher death rate by age group seen in the Chinese population ${ }^{8}$.

The time elapsing between being diagnosed with cancer and found infected with SARS-CoV-2 affected the risk of death, which was all the higher (from 1.6 to 2.7 times higher) the shorter the time. While the differences among pORs for death did not differ significantly, the steadily increasing risk of death associated with the shortening interval between cancer diagnosis and viral infection suggests the need to further explore this clinically relevant point.

Admission to the ICU due to SARS-CoV-2-related disease was not associated with a history of cancer. Among the variables potentially involved in such a situation, some may be related to a patient's underlying health issues and others to differences in the clinical management of patients.

As for any adverse outcomes of SARS-CoV-2-related disease by type or site of primary malignancy, breast cancer and hematological cancers were associated with a higher risk of both hospitalization $(\mathrm{pOR}=2.23$ and 2.0, respectively) and death $(\mathrm{pOR}=3.30$ and 2.39 , respectively). Lung cancer was associated with a fourfold risk of death due to SARS-CoV-2 infection. The clinical importance of such results warrants further investigation, expanding the population considered.

While some clear trends emerged in our findings, some limitations of the study should be mentioned. One lies in that the clinico-epidemiological profile of the population tested changed over the study period, as the viral outbreak developed. During the first fortnight, testing for SARS-CoV-2 was mainly restricted to people presenting with acute (not necessarily complicated) respiratory symptoms and/or reporting having recently traveled in the parts of
China hit by the epidemic. From early March onward, molecular testing was also done on people with contacts potentially at risk. Then, as of mid-March, testing was extended to healthcare professionals and workers in critical areas (police officers, supermarket cashiers and so on). While a selection bias in the population tested cannot theoretically be ruled out, no conditions relating to the clinical history of cancer were assumed in advance and all participants consecutively tested were considered.

In conclusion, this study on 84,246 residents in the Veneto region (northeast Italy)-including 4,789 patients with cancerwho underwent molecular testing for SARS-CoV-2 infection revealed no statistically significant association between a history of malignancy and CV2+ve status. On the other hand, the risk of adverse outcomes of SARS-CoV-2-related disease was significantly higher among patients with cancer, particularly males, older people and those whose cancer had developed no more than 2 years before becoming CV2+ve. Malignancies involving the breast, urinary tract, blood and lung were associated with a higher risk of adverse clinical outcomes of SARS-CoV-2-related disease and/or death.

\section{Methods}

All the 84,246 residents in the Veneto region (Italy) who were consecutively tested for SARS-CoV-2 infection between 22 February and 1 April 2020 (closing date for this study) were considered. No statistical method was used to predetermine sample size. No data were excluded from the analyses. In all cases, the viral infection was sought and confirmed using real-time PCR with reverse transcription and next-generation sequencing. Information on the SARS-CoV-2 status of the population tested was obtained from the archives of the regional health care system up to 15 April 2020. Based on their molecular test results, individuals were classified as SARS-CoV-2-positive (CV2+ve) or SARS-CoV-2-negative (CV2-ve).

The median follow-up for $\mathrm{CV} 2+$ ve patients was $26 \mathrm{~d}$ (interquartile range 21-30 d). The clinical information available in the digital archives of the regional health care system was used to distinguish the clinical outcome of CV2+ve individuals in four main categories by 'level of severity': (1) never hospitalized (that is, in isolation at home); (2) hospitalized at least once (whatever the clinical setting, other than the ICU); (3) admitted to an ICU and requiring invasive ventilation or (4) died. Information on comorbidities was only available for a subgroup of individuals and was therefore not considered.

Based on the data collected by the regional cancer registry, all individuals tested for SARS-CoV-2 infection were distinguished according to their oncological history (people with versus without a history of malignant disease). In detail, those diagnosed with a cancer before 31 December, 2017 were identified from the cancer registry's database, while those diagnosed with a cancer in 2018 and 2019 were identified from the regional databases of hospital discharge records and pathology reports.

For individuals with a history of malignant disease (patients with cancer), the primary cancer site was retrieved from the registry's database together with the interval between the cancer's diagnosis and the date of testing for SARS-CoV-2 infection. For those with multiple cancers the date of diagnosis of the most recent was considered. Patients with cancer were then classified by the time elapsing between their most recent diagnosis of cancer and their testing for SARS-CoV-2 infection $(<2,2-5$ or $>5$ years $)$. 
Statistical analysis. Pearson's chi-square test was used to identify differences in the proportions of individuals between two categories. The Cochran-Armitage test was used to look for any trends for an ordinal variable with more than two categories, and a binary response variable. Univariable and multivariable analyses were conducted by fitting a logistic regression model to the data, and the multivariable models that were fitted included sex and age group $(<70,70-79$, $>80$ years of age). In the multivariable analysis the Wald chi-square statistic was applied to assess the significance of a trend for the interval between cancer diagnosis and infection, and the risk of death. Investigators were not blinded to cancer status of the patients being studied during analysis phase.

The SAS EG v.6.1 (SAS Institute Inc.) statistical package was used for all analyses. All statistical tests were two-tailed. A $P$ value $<0.05$ was considered statistically significant.

Ethics. The study was formally approved by the Bioethics Committee of the Veneto Region, Italy (protocol no. 245343/2020). All personal data concerning individuals involved in this study were managed in a manner consistent with current Italian privacy legislation concerning cancer registries as collectors of personal data for surveillance purposes without any need for explicit individual consent. The descriptive analysis of individual data did not involve any direct or indirect intervention on the population considered ${ }^{15}$.

Reporting Summary. Further information on research design is available in the Nature Research Reporting Summary linked to this article.

\section{Data availability}

The dataset used for the analysis is available at https://doi.org/10.6084/ m9.figshare.12666698.

Received: 25 April 2020; Accepted: 21 July 2020;

Published online: 31 July 2020

\section{References}

1. Lescure, F. X. et al. Clinical and virological data of the first cases of COVID-19 in Europe: a case series. Lancet Infect. Dis. 20, 697-706 (2020).

2. Huang, C. et al. Clinical features of patients infected with 2019 novel coronavirus in Wuhan, China. Lancet 395, 497-506 (2020).

3. Huang, J. F. et al. Fatal outcome in a liver transplant recipient with COVID-19. Am. J. Transpl. 20, 1907-1910 (2020).

4. Ning, L. et al. Novel coronavirus (SARS-CoV-2) infection in a renal transplant recipient: case report. Am. J. Transpl. 20, 1864-1868 (2020).

5. Kamboj, M. \& Sepkowitz, K. A. Nosocomial infections in patients with cancer. Lancet Oncol. 10, 589-597 (2009).

6. van de Haar, J. et al. Caring for patients with cancer in the COVID-19 era. Nat. Med. 26, 665-671 (2020).

7. Matsushita, H. et al. Cancer exome analysis reveals a T-cell-dependent mechanism of cancer immunoediting. Nature 482, 400-404 (2012).
8. Liang, W. et al. Cancer patients in SARS-CoV-2 infection: a nationwide analysis in China. Lancet Oncol. 21, 335-337 (2020).

9. Xia, Y., Jin, R., Zhao, J., Li, W. \& Shen, H. Risk of COVID-19 for patients with cancer. Lancet Oncol. 21, e180 (2020).

10. Yu, J., Ouyang, W., Chua, M. L. K. \& Xie, C. SARS-CoV-2 transmission in patients with cancer at a tertiary care hospital in Wuhan, China. JAMA Oncol. 6, 1108-1110 (2020).

11. Wang, H. \& Zhang, L. Risk of COVID-19 for patients with cancer. Lancet Oncol. 21, e181 (2020).

12. Spicer, J., Chamberlain, C. \& Papa, S. Provision of cancer care during the COVID-19 pandemic. Nat. Rev. Clin. Oncol. 17, 329-331 (2020).

13. Garg, S. et al. Hospitalization rates and characteristics of patients hospitalized with laboratory-confirmed coronavirus disease 2019-COVID-NET, 14 States, March 1-30, 2020. MMWR. Morbid. Mortal. W. 69, 458-464 (2020).

14. Zhang, L. et al. Clinical characteristics of COVID-19-infected cancer patients: a retrospective case study in three hospitals within Wuhan, China. Ann. Oncol. 31, 894-901 (2020).

15. Consiglio dei Ministri. Decreto del Presidente del Consiglio dei Ministri. Gazzetta Ufficiale della Repubblica Italiana 1-80 (3 March 2017).

\section{Acknowledgements}

We thank D. Sacchi, L. Mangone and D. Serraino for their critical discussion of the results of the present study. This work was partly supported by a grant from the Italian Association for Cancer Research (regional grant no. AIRC 6421 to M.R.), and by the Italian Health Ministry's research program 'Performance evaluation and value assessment for cardiovascular and oncological care path in a regional network context: challenges and opportunities', NET-2016-02363853.

\section{Author contributions}

M.R. and M.Z. conceived the study concept and design, interpretation of data and drafted the manuscript. S.G. was responsible for the acquisition and interpretation of data as well as the statistical analysis. All the authors conducted a critical revision of the article for important intellectual content and final approval.

\section{Competing interests}

The authors declare no competing interests.

\section{Additional information}

Supplementary information is available for this paper at https://doi.org/10.1038/ s43018-020-0104-9.

Correspondence and requests for materials should be addressed to M.R.

Reprints and permissions information is available at www.nature.com/reprints.

Publisher's note Springer Nature remains neutral with regard to jurisdictional claims in published maps and institutional affiliations.

(c) The Author(s), under exclusive licence to Springer Nature America, Inc. 2020 


\section{Reporting Summary}

Nature Research wishes to improve the reproducibility of the work that we publish. This form provides structure for consistency and transparency in reporting. For further information on Nature Research policies, see our Editorial Policies and the Editorial Policy Checklist.

\section{Statistics}

For all statistical analyses, confirm that the following items are present in the figure legend, table legend, main text, or Methods section.

n/a Confirmed

\The exact sample size $(n)$ for each experimental group/condition, given as a discrete number and unit of measurement

Х $\square$ A statement on whether measurements were taken from distinct samples or whether the same sample was measured repeatedly

The statistical test(s) used AND whether they are one- or two-sided

Only common tests should be described solely by name; describe more complex techniques in the Methods section.

$\bigotimes$ A description of all covariates tested

Х $\square$ A description of any assumptions or corrections, such as tests of normality and adjustment for multiple comparisons

$\triangle$ A full description of the statistical parameters including central tendency (e.g. means) or other basic estimates (e.g. regression coefficient) AND variation (e.g. standard deviation) or associated estimates of uncertainty (e.g. confidence intervals)

For null hypothesis testing, the test statistic (e.g. $F, t, r$ ) with confidence intervals, effect sizes, degrees of freedom and $P$ value noted Give $P$ values as exact values whenever suitable.

Х $\square$ For Bayesian analysis, information on the choice of priors and Markov chain Monte Carlo settings

$\bigotimes \square$ For hierarchical and complex designs, identification of the appropriate level for tests and full reporting of outcomes

$\triangle \square$ Estimates of effect sizes (e.g. Cohen's d, Pearson's $r$ ), indicating how they were calculated

Our web collection on statistics for biologists contains articles on many of the points above.

\section{Software and code}

Policy information about availability of computer code

Data collection SAS EGv.6.1 (SAS Institute Inc. Cary, NC, USA)

Data analysis SAS EGv.6.1 (SAS Institute Inc. Cary, NC, USA)

For manuscripts utilizing custom algorithms or software that are central to the research but not yet described in published literature, software must be made available to editors and

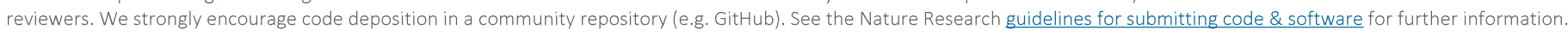

\section{Data}

Policy information about availability of data

All manuscripts must include a data availability statement. This statement should provide the following information, where applicable:

- Accession codes, unique identifiers, or web links for publicly available datasets

- A list of figures that have associated raw data

- A description of any restrictions on data availability

- According to the Journal's requirements, the dataset used for the analyses is available at: https://doi.org/10.6084/m9.figshare.12666698 
Please select the one below that is the best fit for your research. If you are not sure, read the appropriate sections before making your selection.

\ Life sciences

Behavioural \& social sciences

Ecological, evolutionary \& environmental sciences

For a reference copy of the document with all sections, see nature.com/documents/nr-reporting-summary-flat.pdf

\section{Life sciences study design}

All studies must disclose on these points even when the disclosure is negative.

Sample size $\begin{aligned} & \text { Cohort study involving 84,246 consecutive subjects (all resident in the study area, and tested for SARS-CoV-2 infection) during the considered } \\ & \text { time interval (February 22nd } 2020 \text { to April 1st 2020) }\end{aligned}$
Data exclusions The considered cases were consecutive, with no selection (no patients were excluded)
Replication No experiments were performed in this study.
Randomization In this cohort study no allocation to different study arms was performed.
Blinding The type of variables that were analysed (e.g., site of primary cancer) did not allow blinding at analysis level

\section{Reporting for specific materials, systems and methods}

We require information from authors about some types of materials, experimental systems and methods used in many studies. Here, indicate whether each material, system or method listed is relevant to your study. If you are not sure if a list item applies to your research, read the appropriate section before selecting a response.

\begin{tabular}{|c|c|}
\hline$n / a$ & Involved in the study \\
\hline Х & Antibodies \\
\hline Х & Eukaryotic cell lines \\
\hline$\bigotimes$ & Palaeontology and archaeology \\
\hline$\bigotimes$ & $\square$ Animals and other organisms \\
\hline & $\bigotimes$ Human research participants \\
\hline$\bigotimes$ & Clinical data \\
\hline Х & $\square$ Dual use research of concern \\
\hline
\end{tabular}

Methods $\mathrm{n} / \mathrm{a}$ Involved in the study

X $\square$ ChIP-seq

Х $\square$ Flow cytometry

\ $\square$ MRI-based neuroimaging

\section{Human research participants}

\section{Policy information about studies involving human research participants}

Population characteristics

Recruitment

Ethics oversight
Retrospective cohort study involving 84,246 tested for SARS-CoV2 infection. Subjects were distinguished according to age, sex and history of malignancy disease.

The study involved consecutive subjects (all resident in the study area [Italy - Veneto] tested for SARS-CoV2 during the considered time interval (February 22nd to April 1st 2020). No exclusions were applied. No selection bias is expected.

The study project was formally approved by the "Comitato Regionale per la Bioetica Regione del Veneto" i.e.: Bioethics Committee of the Veneto Region- Italy (protocol number: 245343/2020). 